\title{
Model-Order Reduction by First Order Transformations of Linear Networks
}

\author{
M.M. Gourary \\ IPPM, Russian Academy of Sciences, Moscow, Russian Federation, gourary@ippm.ru
}

\begin{abstract}
The paper discusses new methods and algorithms for the reduction of electronic circuits consisting of arbitrary linear components. The approach is based on circuit transformations by successive elimination of nodes including the nodes with inductances and coupled inductances. The suggested elimination algorithms provide first-order accuracy. The passivity criterion of the reduced circuit is presented. It is verified while the process of the elimination. The sequence of eliminations is based on time constants which are evaluated for an arbitrary type of the circuit node. The proposed approach can be considered as an extension of the well-known TICER nodal elimination algorithm for RC circuits. Circuit examples demonstrate the application of the developed approach.
\end{abstract}

Keywords - model order reduction; linear circuits; nodes elimination, transfer function, first order approximation

\section{INTRODUCTION}

Model-order reduction (MOR) of linear interconnection networks is an effective tool to decrease computational efforts in the simulation of integrated circuits (IC). The choice of reduction methods for highdimensional models for circuits with many inputs and outputs is an important issue of this research direction [1]. It should be noted that the use of the most common reduction algorithms based on the Krylov subspace method, for example, PRIMA [2], is faced in this case with significant limitations. These methods were initially developed and studied in detail for the reduction of models with one input and one output ports. When applied to multiport circuits, the number of new vectors at each iteration step is determined by the number of inputs and outputs. Therefore, the dimension of the Krylov subspaces is growing very rapidly, which hampers their application to multiport circuit reduction.

For this reason, the attention of researchers is drawn to the development of alternative approaches, primarily based on selective methods of nodal elimination [3-6]. For example, the popular algorithm TICER [5] performs sequential elimination of circuit nodes, providing a fast reduction of capacitive-resistive interconnects. The required accuracy is achieved by estimating the degree of the smallness by nodal time constant and using it as a criterion for eliminating nodal variables.

For high-dimensional models, the algorithm [5] often does not provide the reduction level that is achieved by projection methods [2] or by methods of balanced implementation [7], but in such cases, the use of selective methods is useful as the preprocessing step [8] to obtain a significant resulting acceleration at subsequent use of more advanced methods.

It is important to note that the TICER allows to reduce RC networks only and therefore the effect of inductances in the network is neglected. However, the growth of working frequencies and/or power of IC makes the contribution of inductances into network response noticeable. It leads to an error when they are neglected. Therefore, an important task is the extension of selective nodal exclusion methods to the generation of reduced model for circuits with small inductance and providing their simulation on the base of the most common Modified Nodal Analysis (MNA).

Currently, several proposals [9-12] are known for considering inductances in node elimination algorithms. The special case of a node with two RL branches and many capacitances is considered in [9], [10]. In [11], the node elimination method is used to obtain high order expressions for the node complex conductivities, and then the optimization method for the synthesis of RLC circuits is used. This approach seems to be unnecessarily complicated and costly. Paper [12] presents the rules for merging two branches of any type attached to an eliminated node. But these rules do not guarantee the first order of accuracy of circuit characteristics, which leads to insufficient accuracy in the case of small inductances. In particular, the proposed rule of merging the LR branch (with a small $\mathrm{L}$ ) and the $\mathrm{C}$ branch is equivalent to discarding the inductance.

In this paper, we assume that inductances in the network have a notable effect on the circuit characteristics, but they are sufficiently small to produce parasitic oscillations defined by second order equations. Thus, the aim of this paper is to develop reduction algorithms based on the elimination method for circuits with small inductances that provide the first order of accuracy.

It can be shown that for a node with a connected inductance, Gaussian elimination of nodal voltage and subsequent neglect of high order terms leads to an equivalent circuit with an asymmetric capacitance matrix. Such circuit does not provide passivity and cannot be used in the circuit simulation. Therefore, we propose the method based on the simultaneous elimination of two circuit variables - the node voltage and inductance current. 
Such a method can be called "inductance elimination" because in the elimination step it removes both node and adjacent inductance. To ensures the first order of accuracy some capacitances are added to a reduced circuit (Section 3 ). The passivity of the obtained scheme is checked by the criterion of positive definiteness of the capacitance matrix. If the eliminated inductance is mutually coupled to another inductance of the circuit (Section 4), then the reduced circuit contains couplings between inductances and capacitances (LC-couplings) which are defined in the paper. It is shown that such couplings result in additional non-zero entries of the MNA capacitance matrix. Therefore, the analysis of a reduced circuit with LCcouplings requires small changes to the simulation program. The rules for the elimination of inductance in the presence of LC-connections were also obtained (Section 5). It is shown that the successive elimination of all inductances in the original circuit leads to an RC circuit without LC connections.

Numerical experiments (Section 7) show that the proposed method provides much smaller errors than the simple exclusion of inductances from the circuit.

\section{ELIMINATION OF RC-NODE BY TICER}

The elimination of RC node [5, 13] (Fig. 1) is based on the expression for star connection transform in the Laplace domain (we label branches by the same indexes as their outward nodes)

$$
\tilde{y}_{i j}=\frac{y_{i} y_{j}}{Y_{n}}=\frac{\left(g_{i}+s c_{i}\right)\left(g_{j}+s c_{j}\right)}{G_{n}+s C_{n}},
$$

Here $y_{i}=g_{i}+s c_{i}, Y_{n}=\sum_{j \in S_{n}} y_{i}=G_{n}+s C_{n}$ are branch and nodal admittances respectively, $S_{n}$ is the set of the indexes of outward nodes of RC-branches connected to the node $n$.
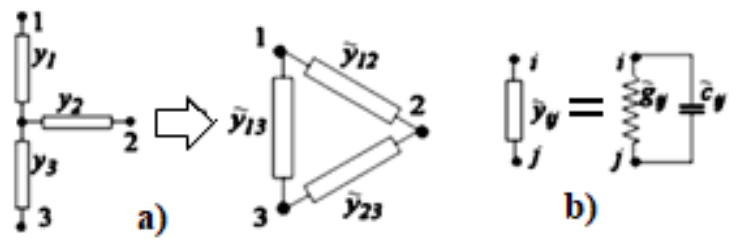

b)

Fig. 1. a) elimination of node with RC-branches only (TICER); b) representation of RC branch

If high order terms of the Laplace variable $s$ in Taylor expansion of (1) are truncated, then one can obtain expressions for conductances and capacitances which must be added to the circuit after node elimination

$$
\text { . } \tilde{g}_{i j}=\frac{g_{i} g_{j}}{G_{n}}, \tilde{c}_{i j}=\frac{g_{i} c_{j}+g_{j} c_{i}}{G_{n}}-\frac{g_{i} g_{j}}{G_{n}^{2}} C_{n}
$$

TICER [5] applies (2) without the last term in $\tilde{c}_{i j}$, and in this case all capacitances of the reduced circuit are positive. Thus, the circuit passivity is provided. However, it has been proved in [14] that despite possible negative capacitances the application of (2) does not lead to the loss of circuit passivity. Hence the exact first order expression (2) can be applied to the nodal elimination to achieve less error.
Numerical experiments show that for circuits with floating nodes (for example, RC lines), the accuracy of the reduced circuit does not improve with an additional negative term. This follows from the following theorem: if each node of the circuit is floating, then the reduction method [5] also provides the first order of accuracy. A special case of this theorem was proved in [6].

\section{INDUCTANCE ELIMINATION}

Here we consider the case of a single inductance connected to RC node (Fig. 2). To obtain the reduced circuit we perform simultaneous elimination of two circuit variables: nodal voltage and inductance current.

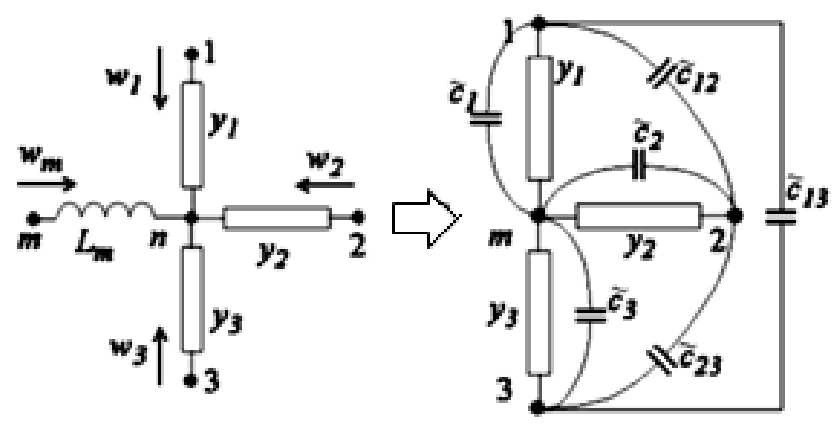

Fig. 2. Elimination of node with inductance branch

Let $w_{\mathrm{i}}$ be the current of $i$-th branch connected to the node $\mathrm{n}$. Taking into account that

$$
w_{i}=y_{i}\left(v_{i}-v_{n}\right) \quad i \in S_{n},
$$

KCL equation for the node $n$ allows us to obtain the inductance current as

$$
w_{m}=-\sum_{j \in S_{n}} w_{j}=-\sum_{j \in S_{n}} y_{j}\left(v_{j}-v_{n}\right) .
$$

The inductance voltage is defined by the equation

$$
v_{n}-v_{m}=s L_{m} w_{m} .
$$

Substituting (4) into (5) we obtain

$$
v_{n}\left(1+s L_{m} Y_{n}\right)=v_{m}-s L_{m} \sum_{j \in S_{n}} y_{j} v_{j} .
$$

The nodal variable derived from (6) by neglecting high order terms is

$$
v_{n}=v_{m}\left(1+s L_{m} G_{n}\right)-s L_{m} \sum_{j \in S_{n}} g_{j} v_{j} .
$$

After substitution of (7) into (3), neglecting high order terms and collecting terms in accordance with nodal voltages we can obtain

$$
\begin{gathered}
w_{i}= \\
y_{i}\left(v_{i}-v_{m}\right)+s \tilde{c}_{i}\left(v_{i}-v_{m}\right)-s \sum_{j \in S_{n}^{i}} \tilde{c}_{i j}\left(v_{i}-v_{j}\right)
\end{gathered}
$$

where $S_{n}^{i}$ is the set $S_{n}$ without index $i$, and

$$
\tilde{c}_{i}=-s L_{m} g_{i} G_{n}, \quad \tilde{c}_{i j}=s L_{m} g_{i} g_{j}
$$

The expression for the current ingoing $i$-th node (8) corresponds to the reduced circuit in Fig. 2:

a) node $n$ is eliminated, and inductance is removed; 
b) the old RC branches are saved in accordance with the first term in (8);

c) new negative capacitances $\tilde{c}_{i}(9)$ are connected to each RC branch representing the second term of (8);

d) new capacitances $\tilde{c}_{i j}$ (9) are inserted between the outward nodes of the RC branches, thereby determining the currents corresponding to the third term (8).

Unlike the case of RC node elimination, the passivity of the circuit after inductance elimination is not secured. So, it is needed to test a passivity condition before the elimination is accepted. It is known that the circuit is passive if its conductance and capacitance matrices are nonnegative definite and the capacitance matrix is symmetric. In our case the conductance matrix remains unchanged and the capacitance matrix is symmetric by the construction. Hence the criterion for symmetric matrix $A$ : $A_{i i} \geq 0,\left|A_{i j}\right| \leq \sqrt{A_{i i} A_{j j}}$ can be applied to the capacitance matrix $C$ to test its non-negative definiteness. It is equivalent to the conditions on branch and nodal capacitances

$$
C_{i}^{N E W} \geq 0,\left|C_{i j}^{N E W}\right| \leq \sqrt{C_{i}^{N E W} C_{j}^{N E W}}, .
$$

Here $c_{i j}^{N E W}=c_{i j}+\tilde{c}_{i j}$. If (10) is not valid for the reduced circuit, then the elimination must be rejected.

The simple example of the inductance elimination is presented in Fig. 3 where conditions (10) for the reduced circuit yield $c<2 L / r^{2}$. This inequality corresponds to the condition of no oscillatory behavior of initial RLC section.

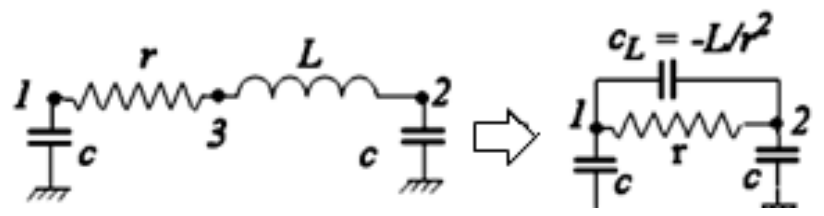

Fig. 3. RLC-section reducing

IV. EliminAtion OF COUPLED INDUCTANCE.

Here we consider the case when the inductance $\mathrm{Ln}$ is coupled with some other inductance $L_{k}$. This coupling is defined by the mutual inductance $L_{n k}$ (Fig. 4).

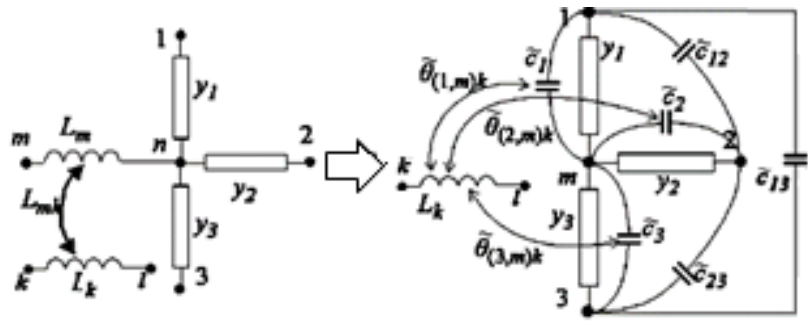

Fig. 4. Elimination of node with coupled inductance branch

In this case $(3,4)$ are valid, and (5) is replaced by

$$
v_{n}-v_{m}=s L_{m} w_{m}+s L_{m k} w_{k} .
$$

and the equation for the voltage of inductance $L_{k}$ must be taken into account:

$$
u_{k}=s L_{k} w_{k}+s L_{n k} w_{m} .
$$

After the elimination of $v_{n}, w_{k}$ we can obtain the first order $\left(O\left(s^{2}\right)\right)$ branch currents and inductance voltages as

$$
\begin{gathered}
v_{n}=v_{m}\left(1+s L_{m} G_{n}\right)-s L_{m} \sum_{j \in S_{n}} g_{j} v_{j}+s L_{m k} w_{k}, \\
w_{i}=\widehat{w}_{i}+s \tilde{\theta}_{(i, m) k} w_{k},
\end{gathered}
$$

where $\widehat{w}_{i}$ is the right hand side of (8), and

$$
\tilde{\theta}_{(i, m) k}=-\tilde{\theta}_{(m, i) k}=L_{m k} g_{i} .
$$

The parameter $\theta$ in (13-15) corresponds to the coupling (Fig. 4) between RC and L-branches (LC-coupling) that can be defined by the following time-domain expressions for the capacitance current $\left(i_{C}\right)$ and inductance voltage $\left(u_{L}\right)$

$$
i_{C}=C \frac{d u_{C}}{d t}+\theta_{L C} \frac{d i_{L}}{d t}, u_{L}=L \frac{d i_{L}}{d t}+\theta_{L C} \frac{d u_{C}}{d t} .
$$

Our notation refers to $k$-th inductance coupled with the capacitance between nodes $m, i$. As far as we know there are no physical devices with such interaction. Formally it can be defined in the context of MNA approach as follows.

MNA conductance and capacitance matrices of RLCK circuit are presented as:

$G^{M N A}=\left[\begin{array}{cc}G & I \\ -I^{T} & 0\end{array}\right], C^{M N A}=\left[\begin{array}{cc}C & \Theta \\ \Theta^{T} & 0\end{array}\right]$.

Here $I$ is the incidence matrix for the circuit inductances.

Matrix $\Theta$ is zeroes for physically realizable RLCK circuits. With the Gaussian elimination entries of $\Theta$ are replaced by nonzero values. Such nonzero values can be defined by nonzero LC-couplings:

$$
\Theta_{i k}=\sum_{j \in S_{i}} \theta_{(i, j) k}, \theta_{(i, j) k}=-\theta_{(j, i) k} .
$$

It is seen from Fig. 4 and (13), (14) that the coupling of eliminated inductance is replaced by LC-couplings with each RC-branch of eliminated node.

Due to the symmetry of $C^{\mathrm{MNA}}$ (14) the passivity condition can be presented in the form

$$
C_{i}^{N E W} \geq 0,\left|\Theta_{i k}\right| \leq \sqrt{L_{k} C_{i}},\left|\Theta_{i k}\right| \leq \sqrt{L_{k} C_{j}} .
$$

Standard SPICE-like simulators cannot process LCcouplings but this capability can be easily implemented taking into account corresponding time-domain expressions (16) and MNA capacitance matrix (17).

\section{ELIMINATIONS UNDER LC COUPLINGS}

The possible occurrence of LC-couplings in the reduced circuit requires to analyze the case when eliminating inductance has LC-coupling with any RCbranch (Fig. 5). 


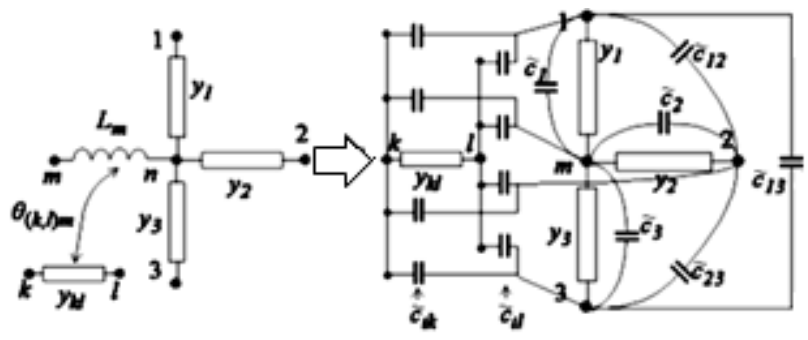

Fig. 5. Elimination of node with LC-coupled branch

In this case (5) should be replaced by

$$
v_{n}-v_{m}=s L_{m} w_{m}+s \theta_{(k, l) m}\left(v_{k}-v_{l}\right) .
$$

After transformations like (6-8) we get

$$
\begin{gathered}
w_{i}=\widehat{w}_{i}+s g_{i} \theta_{(k, l) m}\left(v_{k}-v_{l}\right)= \\
\widehat{w}_{i}+s g_{i} \theta_{(k, l) m}\left(v_{k}-v_{i}\right)+s\left(-g_{i} \theta_{(k, l) m}\right)\left(v_{l}-v_{i}\right) .
\end{gathered}
$$

The current flowing into the node $m$ is defined as

$$
w_{m}=-\sum_{j \in S_{n}} \widehat{w}_{j}+s\left(-G_{n} \theta_{(k, l) m}\right)\left(v_{k}-v_{m}\right)+
$$$$
s G_{n} \theta_{(k, l) m}\left(v_{l}-v_{m}\right) \text {. }
$$

The last terms in $(21,22)$ represent currents through capacitors which values are

$$
\tilde{c}_{k i}=-\tilde{c}_{l i}=g_{i} \theta_{(k, l) m}, \quad \tilde{c}_{l m}=-\tilde{c}_{k m}=G_{n} \theta_{(k, l) m} .
$$

Thus, after the elimination of the capacitance-coupled inductance the reduced circuit is supplemented by capacitances (23) shown in Fig. 5.

The special case of the elimination can be considered when the inductance is LC-coupled with one of adjacent RC branches. The resulting circuit contains only one additional capacitance $\tilde{c}_{i}^{\prime}=g_{i} \theta_{(n, l) m}$ connected to the coupled branch (besides capacitances defined by (9)). This case is shown in Fig. 6.

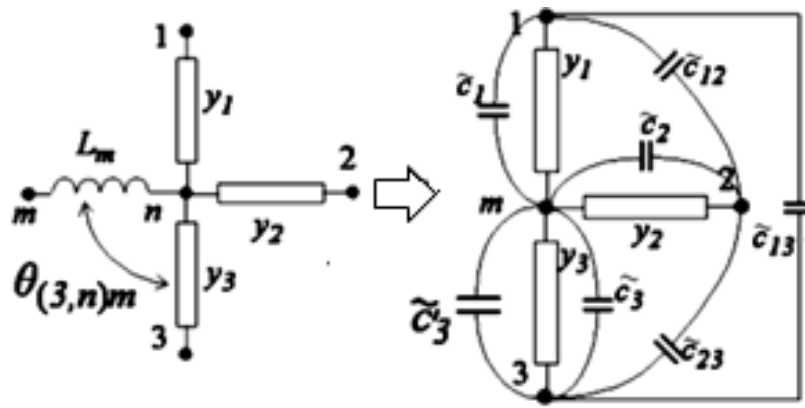

Fig. 6. Elimination of the inductance LC-coupled with the adjacent RC branch

Note that if all inductances of the circuit are sequentially eliminated, then the reduced RLCK circuit is transformed to an RC circuit. The example of the reduction of two coupled LR-branches $\left(L_{1}, r_{1}\right.$ and $\left.L_{2}, r_{2}\right)$ is presented in Fig. 7. The full elimination of both inductances leads to RC-circuit with capacitances $c_{1}=L_{1} / r_{1}^{2}, c_{2}=L_{2} / r_{2}^{2}$, $c_{M}=L_{12} / r_{1} r_{2}$.

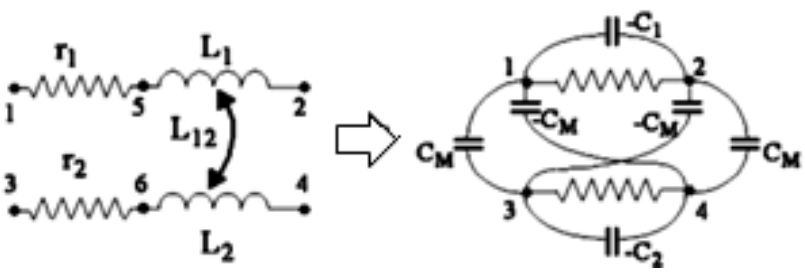

Fig, 7. The result of successive inductance eliminations in the circuit of coupled LR branches

LC-couplings also must be taken into account in RCnode elimination $(1,2)$. If $m$-th RC-branch of eliminating node $n$ is LC-coupled with $k$-th inductance (Fig. 7, $m=1$ ), then term $\theta_{(m, n) k} \cdot$ Wk must be added to $m$-th current (3) and to KCL for node $n$. After the elimination of $v_{n}$ and neglecting high order terms we obtain

$w_{i}=\sum_{j \in S_{n}^{i}}\left(\tilde{g}_{i j}+s \tilde{c}_{i j}\right)\left(v_{i}-v_{j}\right)-s \theta_{(m, n) k} \frac{g_{i}}{G_{n}} w_{k}$,

$w_{m}=\sum_{j \in S_{n}^{m}}\left(\tilde{g}_{m j}+s \tilde{c}_{m j}\right)\left(v_{m}-v_{j}\right)+\theta_{(m, n) k} \frac{G_{n}-g_{m}}{G_{n}} w_{k}$.

Here $\tilde{g}_{i j}, \tilde{c}_{i j}$ are defined by (2), and last terms in (24, 25) correspond to LC-couplings in the reduced circuit (Fig. 7) $\tilde{\theta}_{(m, i) k}=\theta_{(m, n) k} \frac{g_{i}}{G_{n}}$. Thus, after node elimination the branch coupling parameter is distributed among new branches adjacent to the node $\mathrm{m}$ in proportion to their conductances.

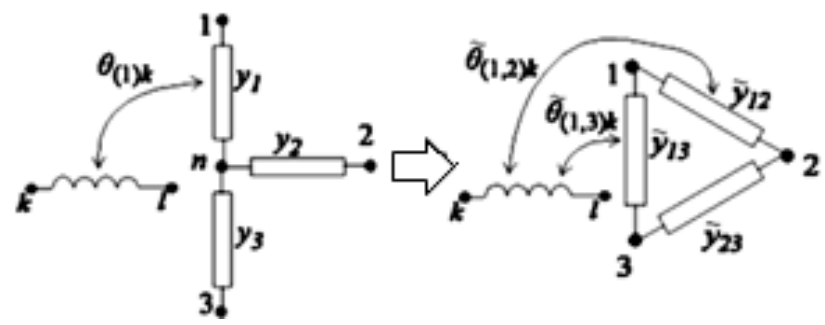

Fig. 8. Elimination of RC-node in the presence of LCcoupling in one of branches

\section{ELIMINATION PROCESS}

The algorithm performs the next nodal/inductance elimination if a sufficiently small error in the transfer characteristics of the reduced circuit is provided. The error of a first-order method is determined by neglected terms of higher orders. For the case of eliminating an RC node (2), it is possible to estimate the term of the $m$-th order [6] in the form $\delta_{\mathrm{m}}=\mathrm{O}\left(\mathrm{s}\left|\tau_{\mathrm{n}}\right|^{\mathrm{m}}\right)$, where $\tau_{n}=\tau_{n}^{R C}=C_{n} / G_{n}$ is the RC time constant of the $n$-th node. Therefore, the excluded nodes are selected [5] in accordance with increasing values of their time constant until the condition $\tau_{n}<\tau_{\min }$ is true. Hhere $\tau_{\min }$ is estimated by the maximum operating frequency of the device $-\tau_{\min }=a / f_{\max }$. The coefficient $a$ is determined by the trade-off between accuracy and the reduction coefficient of the circuit.

To eliminate inductances, it is necessary to determine the nodal time constants for nodes with inductance. The neglected $m$-th order terms, in $(7,8)$, can be estimated as $\delta_{m}^{n}=O\left(\left|s \tau_{n}^{R C}\right|^{k}\left|s \tau_{n}^{R L}\right|^{l}\right),(r+l=m) \quad$ where $\tau_{n}^{R C}$ is defined above and $\tau_{n}^{R L}=L G_{n}$. Therefore, the nodal time 
constant in this case can be evaluated as the worst-case value $\tau_{n}=\max \left(\tau_{n}^{R C}, \tau_{n}^{L R}\right)$. At each step, the node to be eliminated is determined by the minimum time constant among all the nodes of the circuit with no more than one connected inductance.

In the presented analysis we ignored the nodes with multiple inductances. But this case does not require a special analysis because presented algorithms are enough to provide the elimination of all small inductances. It can be explained by the following way. We do not consider DC undefined circuits containing inductance loops. Consequently, each connected graph of inductances is a tree that always has leaf nodes with only one connected inductance (Fig. 9). Therefore, if all inductances of the tree are sufficiently small then single-inductance algorithms will sequentially eliminate them.

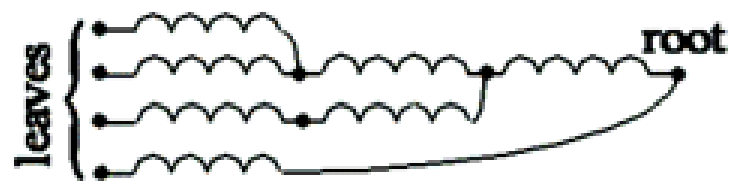

Fig. 9. The tree of the inductances. Each leave node has only one connected inductance

VII. NUMERICAL EXAMPLES

Two circuit examples were simulated to test proposed inductance elimination algorithms. The first example is RLC line containing ten identical sections (Fig. 3) with parameters $r=1 \Omega, \mathrm{L}=0.4 \mathrm{pH}, \mathrm{C}=1 \mathrm{pF}$. Note that inductance value is slightly less than the value providing the passivity condition $\left(L_{\max }=0.4 \mathrm{pH}\right)$.

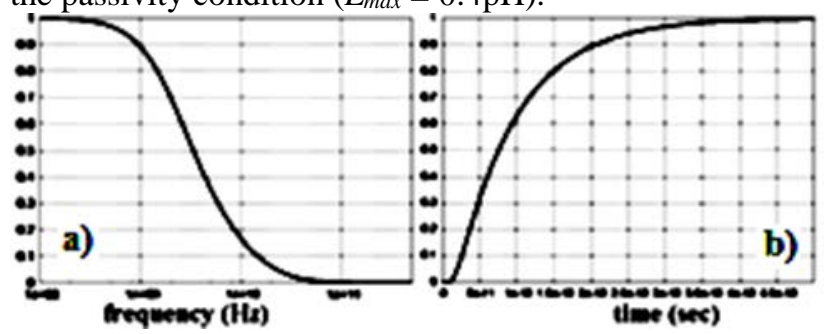

Fig. 10. RLC line responses in the frequency (a) and time (b) domains

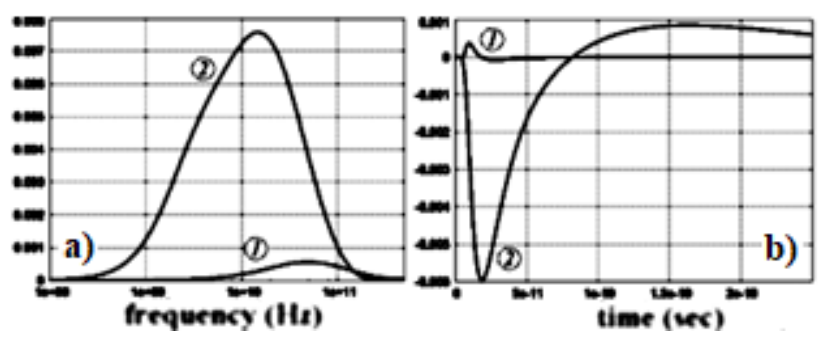

Fig. 11. Elimination (1) and the neglect (2) errors of RLC line in the time (a) and frequency (b) domains

Errors of two reduced circuits were compared:

- RC line obtained by the elimination of all inductances using the proposed method;
- RC line using the neglect of all inductances.

The frequency- and time-domain responses of the original RLC line (Fig. 10) as well as the responses of two reduced circuits were computed. The time-domain simulation was performed under the unit step excitation with $t_{\text {rise }}=3$ 3psec. The reduction error for each method was evaluated as the difference between the responses of the original and reduced circuits (Fig. 11). One can see that the elimination error is about tenfold less than the neglect error both in frequency- domain and time-domains.

The second example presents inductively coupled branches (Fig. 7) with load capacitances (Fig. 12). The reduced circuit is obtained in accordance with Fig. 7. Output frequency responses are shown in Fig. 10a. Timedomain crosstalk waveforms under input pulse with unit magnitude and $t_{\text {pulse }}=20 \mathrm{psec}, t_{\text {rise }}=1$ psec, $t_{\text {fall }}=4$ psec are presented in Fig. 10b. Note that in spite of large errors in the frequency response of the reduced circuit at $\mathrm{f}>$ $100 \mathrm{MHz}$ the error in the time domain crosstalk waveform is sufficiently small. It is important to point out that neglect of inductances in the circuit results in zero crosstalk waveforms under any input excitation.

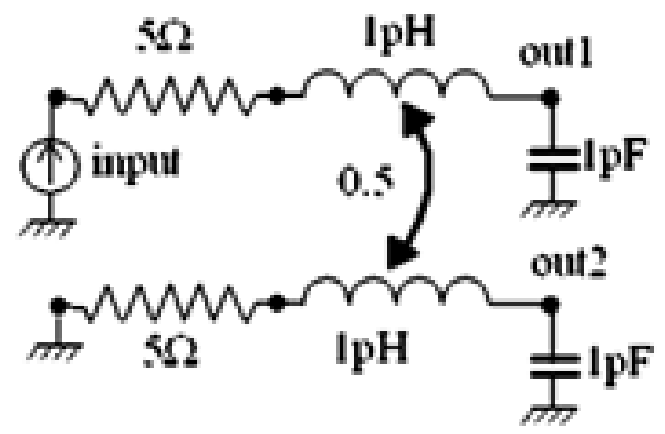

Fig. 12. Coupled RL-branches with capacitance loads

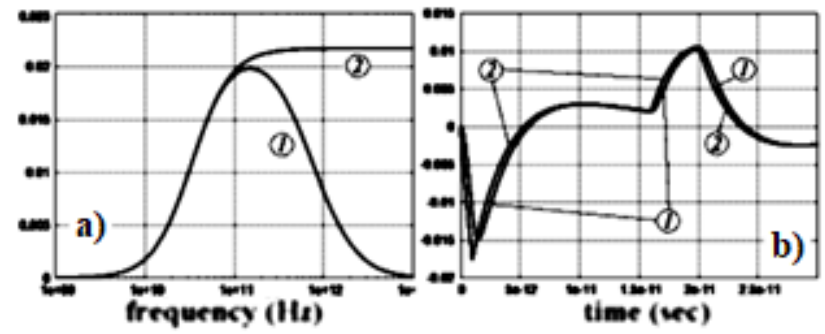

Fig. 13. Crosstalk frequency (a) and time-domain (b) responses in inductively coupled branches

\section{CONCLUSION}

New approach to construct selective MOR algorithms for an arbitrary RLCK circuits are presented in the paper. Proposed method is based on the sequential elimination of circuit nodes. It supplements well-known TICER method with elimination algorithm for connected inductance nodes.

The elimination of one of mutually coupled inductances results in including new component inductance-capacitance coupling into the reduced circuit. To perform the simulation of such circuit minor corrections in the circuit simulator are required. The complete 
elimination of all inductances results in RC circuit. The important advantage of the method is preserving the first order accuracy of the reduced transfer functions, so the method is effective for circuits with small inductances. The passivity condition of the reduced circuit is derived. The condition can be easily verified at each elimination step to accept or reject inductance elimination.

Similarly with TCER method the sequence of eliminations is defined by minimal nodal time constant and user-defined stopping criterium is applied. For nodes with inductances expressions for time constants are derived in the paper.

Circuit examples presented in the paper demonstrate essential decrease of approximation errors under application of proposed approach in the comparison with reducing by total neglect of circuit inductances.

\section{REFERENCES}

[1] Schilders Wil H. A., The Need for Novel Model Order Reduction Techniques in the Electronics Industry // In "Model Reduction for Circuit Simulation", Series: Lecture Notes in Electrical Engineering (LNEE). Vol. 74, Springer, 2011, pp. 3-23.

[2] Odabasioglu, C. and Pileggi, PRIMA: passive reducedorder interconnect macromodeling algorithm, 1997 Proceedings of IEEE International Conference on Computer Aided Design (ICCAD), San Jose, CA, USA, 1997, pp. 58-65.

[3] Gurarij M. M., Rusakov S. G. Sintez makromodelej fragmentov BIS metodom vozmushchenij // Mikroelektronika. - 1977. - № 5. - C. 406-409,

[4] Gourary M M., Zharov M.M., Rusakov S.G., Ul'yanov S.L., Metody vozmushchenij i selektivnye metody $\mathrm{v}$ zadachah redukcii vysokorazmernyh modelej/ Sbornik trudov III Vserossijskoj nauchno-tekhnicheskoj konferencii «Problemy razrabotki perspektivnyh mikro- i nanoelektronnyh sistem». - M: IPPM RAN - 2008. pp. 8691.
[5] Sheehan, B.N.: "TICER: Realizable reduction of extracted RC circuits," 1999 IEEE/ACM International Conference on Computer-Aided Design. Digest of Technical Papers (Cat. No.99CH37051), San Jose, CA, USA, 1999, pp. 200-203.

[6] Sheehan, B.N.: Realizable Reduction of RC Networks, in IEEE Transactions on Computer-Aided Design of Integrated Circuits and Systems, vol. 26, no. 8, pp. 13931407, (2007).

[7] Moore, B. C.: Principal component analysis in linear systems: Controllability, observability andmodel reduction. IEEE Trans. Autom. Control, vol. AC-26, no. 1, pp. 17-32, (1981).

[8] Zharov M.M., Rusakov S.G. Dvuhurovnevaya redukciya modelej parazitnyh cepej vysokogo poryadka, V sbornike trudov Vserossijskoj nauchno-tekhnicheskoj konferenci "Problemy razrabotki perspektivnyh mikroelektronnyh sistem - 2006" (MES-2006), Moskva, IPPM RAN, 2006, pp. 87-90.

[9] Sheehan, B.N.: Branch merge reduction of RLCM networks," ICCAD-2003. International Conference on Computer Aided Design (IEEE Cat. No.03CH37486), San Jose, CA, USA, pp. 658-664 (2003).

[10] Rong, J, Chen, C.C.-P.: Realizable reduction for electromagnetically coupled RLMC interconnects. In: Proc. Design, Automation and Test in Europe Conf., vol. 12 pp. 1400-1401, (2004).

[11] Qin, Z., Cheng, C-K,: Realizable parasitic reduction using generalized Y-d transformation. In: Proc. IEEE/ACM Design Automation Conference, Anaheim, pp. 220-225, (2003).

[12] Amin, C.S., Chowdhury, M.H., Ismail, Y.I.: Realizable Reduction of Interconnect Circuits Including Self and Mutual Inductances. IEEE Trans. Computer-Aided Design of Int. Circ.Syst., vol. 24, no. 2, pp. 271 - 277, (2005).

[13] Network reduction by inductance elimination, Model Reduction for Circuit Simulation . Model Reduction for Circuit Simulation, Series: Lecture Notes in Electrical Engineering (LNEE), Vol. 74, Springer, 2011, pp. 149-161.

[14] Zuochang, Ye, Vasilyev, Dmitry, Zhenhai, Zhu, Phillips, Joel R.: Sparse Implicit Projection (SIP) for reduction of general many-terminal networks. In: Proc. Int. Conf. Computer-Aided Design, San Jose, pp. 736-743, (2008) 